\title{
Biological Control of Aflatoxin and Cyclopiazonic Acid Contamination of Peanuts
}

\author{
Richard J. Cole and Joe W. Dorner
}

\begin{abstract}
Abstruct
Aflatoxin contamination of peanuts, which results from infection and growth by Aspergillus flavus and $A$. parasiticus, is a serious economic problem in developed countries and a potentially serious human and animal health problem in developing countries. Cyclopiazonic acid is another mycotoxin produced by $A$. flavus that has been found as a contaminant of peanuts. A biological control strategy has been developed for reducing contamination of peanuts by these toxins. In involves inoculation of fields with competitive, non-toxigenic strains of the same fungi. The methodology has been tested for several years and has produced reductions in aflatoxin ranging from 30 to $90 \%$. Field studies conducted in 1997 yielded an aflatoxin reduction of $91.6 \%$ and a reduction in cyclopiazonic acid contamination of $85.7 \%$.
\end{abstract}

Key words : aflatoxin, cyclopiazonic acid, peanut

\section{Introduction}

Aflatoxins are potent hepatotoxic, carcinogenic compounds produced by Aspergillus flavus Link: Fr. and Aspergillus parasiticus Speare ${ }^{1)}$. Cyclopiazonic acid (CPA) is another potent mycotoxin that is produced by $A$. flavus, but not by $A$. parasiticus. When these fungi invade and grow in commodities such as peanuts, corn, cottonseed, and tree nuts, the resulting contamination with the aflatoxins and CPA often makes the commodity unfit for consumption. The problem in developed countries is primarily an economic problem which results from extensive manufacturing processes that result in removal of these mycotoxins from the food supply. However, in developing countries the problem is a human and animal health problem, particularly in areas with high exposure to hepatitis B virus.

Because peanuts in the U.S.A. are used primarily for food, and the major portion of the crop is consumed as peanut butter and the major sector of the population that consumes peanut butter is the children, strict regulatory limits for the amount of aflatoxin allowable in finished peanut products have been established. Although the United States Food and Drug Administration has an action level of $20 \mathrm{ppb}$ of total aflatoxins in food products, international tolerances for aflatoxin are much lower, typically in the range of $0-4 \mathrm{ppb}$.

Aflatoxin contamination of peanuts can occur during postharvest curing and storage, however, the most significant contamination usually occurs prior to harvest during periods of late-season drought stress as peanuts are maturing. The only known method for controlling preharvest aflatoxin contamination in peanuts is proper irrigation, an option that is

USDA, ARS, National Peanut Research Laboratory, Dawson, GA 31742, USA 
unavailable to the majority of peanut growers.

Cyclopiazonic acid is another important mycotoxin known to be produced by a variety of important fungi including $A$. flavus. In addition, CPA has been found as a natural contaminant of corn and peanuts, often occurring together with the aflatoxins ${ }^{4,6}$. It was implicated as the causative agent in a human intoxication involving consumption of contaminated millet ${ }^{5}$. With the discovery of CPA production by $A$. flavus, 54 isolates of $A$. flavus were investigated for production of CPA and aflatoxin ${ }^{3)}$. It was found that 28 of the 54 isolates (52\%) produced CPA whereas only 18 isolates (33\%) produced aflatoxin. Regulatory limits for CPA have not been established; however; because of the likely co-occurrence of aflatoxin and CPA in commodities, efforts to attain biological control of aflatoxin need to attain control of CPA as well.

Basic studies into the mechanism(s) involved in preharvest aflatoxin/CPA contamination conducted over the years have provided insight into possible strategies for prevention/ control. Based on information obtained from these studies, several strategies have been investigated. The most promising strategies found to date are development of resistant varieties (drought tolerant varieties) and biological control. The most advanced strategy developed to date is the use of biological control which is based on the concept of biocompetetive exclusion. agents for control of both preharvest and postharvest aflatoxin/ CPA contamination. Normally, when peanuts are exposed to late-season drought stress, populations of wild strains of $A$. flavus and $A$. parasiticus build up to high levels, and the opportunity for invasion and contamination of peanuts is high. With the current biocontrol strategy, a high population of non-toxigenic, but competitive strains of $A$. flavus and $A$. parasilicus are introduced into the soil early in the growing season to occupy the niche that would normally be occupied by the native mostly toxigenic strains (ratio of 19 toxigenic to 1 non-toxigenic). By the time peanuts are exposed to late-season drought (conditions necessary for aflatoxin contamination), populations of the applied, biocompetitive strains are at higher levels than the native, toxigenic strains ( 1 toxigenic to 200 non-toxigenic) and thus have a distinct competitive advantage in invading and contaminating peanuts developing in the soil.

\section{Formulations}

Various methods and formulations have been used to apply the biocompetitive fungi to the soil. In earliest studies, fungi were grown in liquid culture and homogenized. The homogenates were sprinkled over peanut plants to distribute conidia and fungal mycelium. Most studies have utilized rice as a carrier of the biocompetitive fungi. Rice inoculum was prepared by culturing each strain on autoclaved, long-grain rice in $2800 \mathrm{ml}$ Fernbach flasks ( $500 \mathrm{~g}$ of rice with $150 \mathrm{ml}$ distilled water). Rice was inoculated with $1 \mathrm{ml}$ of a conidial suspension $\left(10^{6} / \mathrm{ml}\right)$ and incubated at $30^{\circ} \mathrm{C}$ for 4 days on a rotating platform $(2 \mathrm{rev} / \mathrm{min})$ tilted $70^{\circ}$ from horizontal to gently agitate the rice and prevent fungal sporulation. Rice inoculum was then dried in a shallow pan in a forced-air draft oven at $50^{\circ} \mathrm{C}$ for 6 hours or until the moisture content was equal to or less than $7 \%$. Rice inoculum was stored at $5{ }^{\circ} \mathrm{C}$ until used.

Because the solid-state fermentation formulation could not be economically and feasibly scaled-up to manufacturing scale, studies were conducted to develop an effective but more economical formulation. A newly developed formulation (patent pending) based on impregnation of the substrate (rice or other suitable grain) with conidia of the biocompetitive fungi 
Table 1 Aflatoxin and CPA contamination (ppb) of field-grown peanuts in 1997 treated with a combination of biocompetitive agents including NRRL 21882 (A. flavus) and NRRL 21369 (A. parasiticus color mutant).

\begin{tabular}{lrrrrr}
\hline \hline \multirow{2}{*}{ Treatment } & \multicolumn{3}{c}{ Aflatoxin } & & CPA \\
\cline { 2 - 3 } \cline { 6 - 6 } & Edible & Inedible & Total Kernels & & Total Kernels \\
\hline Control & 22.2 & 3643.9 & 603.5 & & 30.7 \\
Treated & 2.5 & 290.5 & 50.8 & & 4.4 \\
\% Reduction & 88.7 & 92.0 & 91.6 & & 85.7 \\
\hline
\end{tabular}

Values are the mean of 8 samples averaging 7 pounds each.

appears to be as effective as solid-state fermentation, but is considerably more practical and economical than solid-state fermentation.

For field application, the formulation was placed in a Gandy box fitted to a tractor and banded over the peanut row, optimally at 40-45 days after planting. At this point the width of a peanut row (measured from the outer edges of the foliage canopy) is generally about 18 inches. The inoculum filters through the canopy of foliage and comes to rest in a humid, protected environment on the soil surface under the canopy. Uptake of moisture by the inoculum granules results in growth of the incorporated biocompetitive fungi, which produces an abundance of conidia on the surface of the granules. As the conidia are dispersed over the soil surface, full delivery of the biocompetitive fungi is realized.

\section{Biocompetetive Exclusion Studies}

Various non-toxigenic strains of $A$. flavus and $A$. parasiticus have been tested for purposes of biological control. Initial studies, begun in 1987, utilized a strain of naturally occurring $A$. parasiticus (NRRL 18991) that was effective in reducing aflatoxin contamination ${ }^{2)}$. However, this strain was subsequently found to be a producer of $\mathrm{O}$ methylsterigmatocystin, the immediate biosynthetic precursor to aflatoxin $B_{1}$. Because this compound is a suspected carcinogen, efforts were undertaken to find strains that were not only non-aflatoxigenic, but also not capable of producing other toxins or other metabolites that were suspected mutagens, carcinogens, or the like.

Strains used in more recent studies include: A. flavus (NPL 45, NRRL 21882), a strain isolated from a peanut at NPRL in 1991 that does not produce aflatoxin, CPA, or known intermediates in the aflatoxin biosynthetic pathway (some of which are toxic or carcinogenic) ; A. flavus (NRRL 21368), a UV-induced color mutant of NPL 45 ; A. parasiticus (NRRL 21369), a UV-induced color mutant of NRRL 6111, an aflatoxigenic strain that had been shown in earlier studies to be competitive under field conditions. Results from these studies (plot and field studies) resulted in reductions of the aflatoxins ranging from $30-90 \%$. The results varied depending on effectiveness of the various strains tested. Results from field studies conducted in 1997 utilizing our most advanced strains are presented in Table 1. The average reduction in aflatoxin in treated peanuts (total kernels) compared to controls was $91.6 \%$, and the average reduction in CPA in treated peanuts compared to controls was $85.7 \%$.

Efficacy studies were continued in 1998 and 1999. These studies will examine the combined effects of biocontrol and the more aflatoxin/CPA "resistant" peanut varieties. In 
addition, other non-toxigenic species of Aspergillus (koji molds), which have been used safely in the food industry for many years, are being tested for their ability to reduce aflatoxin/CPA contamination.

Studies have also been conducted, and will be continued, to determine if treatment with these competitive fungi can reduce aflatoxin contamination that occurs during storage. Preliminary results were very promising. Other work devoted to the final development of more effective and more economical formulations will be continued.

Thus, biocontrol studies over the last 12 years have shown that biocompetitive exclusion using non-toxigenic, but competitive strains of $A$. flavus and $A$. parasiticus, provides a very effective and economic strategy to reduce the levels of preharvest and postharvest aflatoxin contamination levels in peanuts and possibly other susceptible crops.

\section{References}

1) CAST. (1989) Mycotoxins: Economic and Health Risks. Council for Agricultural Science and Technology, 137 Lynn Avenue, Ames, IA 50010. 91 pp.

2) Dorner, J. W., R. J. Cole, and P. D. Blankenship. (1992) Use of a biocompetitive agent to control preharvest aflatoxin in drought stressed peanuts. J. Food Prot. $55: 888-892$.

3) Gallagher, R. T., J. L. Richard, H. M. Stahr, and R. J. Cole. (1978) Cyclopiazonic acid production by aflatoxigenic and non-aflatoxigenic strains of Aspergillus flavus. Mycopathologia 66:31-36.

4) Lansden, J. A., and J. I. Davidson. (1983) Occurrence of cyclopiazonic acid in peanuts. Appl. Environ. Microbiol. 45 : 766-769.

5) Rao, L. B., and A. Husain. (1985) Presence of cyclopiazonic acid in kodo millet (Paspalum scrobiculatum) causing 'kodua poisoning' in man and its production by associated fungi. Mycopath ologia 89 : 177-180.

6) Urano, T., M. W. Trucksess, R. W. Beaver, D. M. Wilson, J. W. Dorner, and F. E. Dowell. (1992) Co-occurrence of cyclopiazonic acid and aflatoxins in corn and peanuts. J. AOAC Int. 75 : 838-841. 